\title{
Avoiding noise frequency interference with binary phase pulse driving and CDS for capacitive TSP controller
}

\author{
Yong-Sung Ahn, Taek-Joon Ahn, Kyongsu Lee, and Jin-Ku Kanga) \\ Dept. of Electronics Engineering, Inha University, \\ 100 Inha-ro, Nam-gu, Incheon 402-751, Republic of Korea \\ a) jkang@inha.ac.kr
}

\begin{abstract}
Noise frequency interference avoidance (NFIA) using binary phase pulse driving and correlated double sampling (CDS) circuit technique is applied to avoid the interference of noise signal in capacitive touch screen panel (TSP). The proposed analog front-end circuitry is composed of a charge transfer circuit and a two-stage cascaded CDS circuit. The purpose of the two-stage cascaded CDS circuit is to remove harmonic noise frequencies interfering with touched data in TSP. If the system detects that noise frequencies interfere with the touched data, the proposed NFIA technique can be applied. Our proposed methodology is implemented in a real TSP system using $0.13-\mu \mathrm{m}$ CMOS process and the measured SNR is $46 \mathrm{~dB}$ with the scan rate of $120 \mathrm{~Hz}$ in 21.5 inch TSP.
\end{abstract}

Keywords: noise frequency interference avoidance (NFIA), binary phase pulse signaling, correlated double sampling (CDS), touch screen panel (TSP) Classification: Integrated circuits

\section{References}

[1] J.-H. Yang, S.-H. Park, J.-M. Choi, H.-S. Kim, C.-B. Park, S.-T. Ryu and G.-H. Cho: ISSCC Dig. Tech. Papers (2013) 390. DOI:10.1109/ISSCC.2013.6487783

[2] C. C. Enz and G. C. Temes: Proc. IEEE 84 (1996) 1584. DOI:10.1109/5.542410

[3] M. Hamaguchi, A. Nagao and M. Miyamoto: ISSCC Dig. Tech. Paper (2014) 214. DOI:10.1109/ISSCC.2014.6757405

\section{Introduction}

Recently, touch screen panel (TSP) using a finger or a stylus pen is popular in tablet PCs and smart phones. Touched finger is for selecting icons, scrolling text on webbrowser, and drawing a picture on the display panel. In desktop PC users use input devices as keyboard and mouse. But, TSP quickly replaces keyboard and mouse as input devices in mobile devices. Input devices such as keyboard and mouse is not critical with $60 \mathrm{~Hz}$ noise and noise caused by a lamp or a charger. However, in TSP 
various electrical noises interfere with the touched data [1]. Therefore a technique to avoid these noise interferences with normal touched data should be implemented.

In this paper we proposed a noise frequency interference avoidance (NFIA) methodology to protect the touched data in capacitive touch screen panels. The $60 \mathrm{~Hz}$ power line noise and the lamp noise can be easily removed by the proposed AFE circuitry. But, the charger noise exists widely as in-band and out-of-band noise. The in-band noise can be removed digital filtering method. But, it is difficult to remove out-of-band noise caused from the charger noise and other harmonics noises from touched data. Our proposed technique can remove the out-of-band noise and the harmonics noise. In this paper, first, we describe the proposed analog front-end circuitry. Secondly, explain the technique for reducing noise frequency interference effect. Finally, the measured results will be given.

\section{Proposed architecture}

The proposed analog front-end circuitry in the receiver $(\mathrm{Rx})$ for capacitive touch screen panel is composed of a differentiator, a charge transfer (CT) circuit and twostage cascaded correlated double sampling (CDS) circuit. Depending on noise conditions one CDS or two CDS circuits are used. The circuitry is shown in Fig. 1. The CT circuit is transferring the charge in TSP capacitor to the internal capacitor $\left(\mathrm{C}_{\text {st } 1}\right)$. The $1^{\text {st }}$ and $2^{\text {nd }}$ stage CDS circuit are for reshaping the stored charge in $\mathrm{C}_{\text {st1 }}$ to suppress DC noise and harmonic frequency noise in TSP.

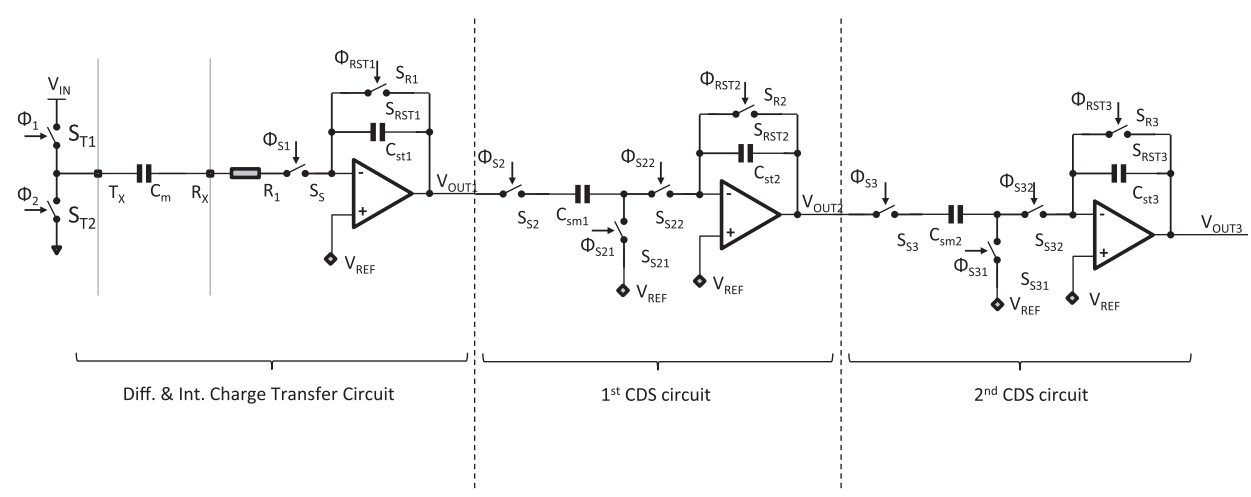

Fig. 1. Proposed analog front-end circuitry in the receiver

In the CT circuit, using $\mathrm{S}_{\mathrm{T} 1}$ or $\mathrm{S}_{\mathrm{T} 2}, \mathrm{C}_{\mathrm{m}}$ is charged or discharged. If $\mathrm{S}_{\mathrm{T} 1}$ is on, the current is flowing from $V_{I N}$ node to $R_{1}$ and $C_{m}$ is charged. Then $S_{S}$ is to sample the voltage signal and $\mathrm{C}_{\mathrm{st} 1}$ integrates the sampled signal in the $\mathrm{CT}$ circuit. Correlated double sampling technique is to reduce DC noise and 1/f noise [2]. Also, CDS technique can be used to reduce even harmonics of the operating clock frequency in TSP. However, odd harmonics of the operating frequency cannot be removed. In order to reduce interference caused by harmonics of the operating frequency, it is widely used that the frequency hopping $(\mathrm{FH})$ methodology in electronic sensing nodes. But, sometimes the hopping frequency to avoid noise interference causes another unwanted frequency interference to other blocks in system. 
Our proposed noise frequency interference avoidance technique adopts a binary phase pulse driving in the transmitter (Tx) with CDS circuitry. The binary phase pulse driving in Tx reduces the frequency interference by modifying the driving signal phase without changing the signal frequency in the Tx. The proposed noise frequency interference avoidance algorithm takes either the normal RZ (Return Zero) pulse driving in Tx with a single stage CDS in Rx or the binary phase pulse driving in Tx with two-stage cascaded CDS in Rx depending on noise frequency interference conditions. Fig. 2 shows timing diagram operating two modes (mode-1: $\mathrm{RZ}$ pulse driving $+1^{\text {st }} \mathrm{CDS}$ operation and mode-2: binary phase pulse driving $+1^{\text {st }}$ $\& 2^{\text {nd }}$ CDS operation) of analog front-end circuitry in Rx. The $n$-time pulsing and integration is executed and then the final symbol value is averaged.

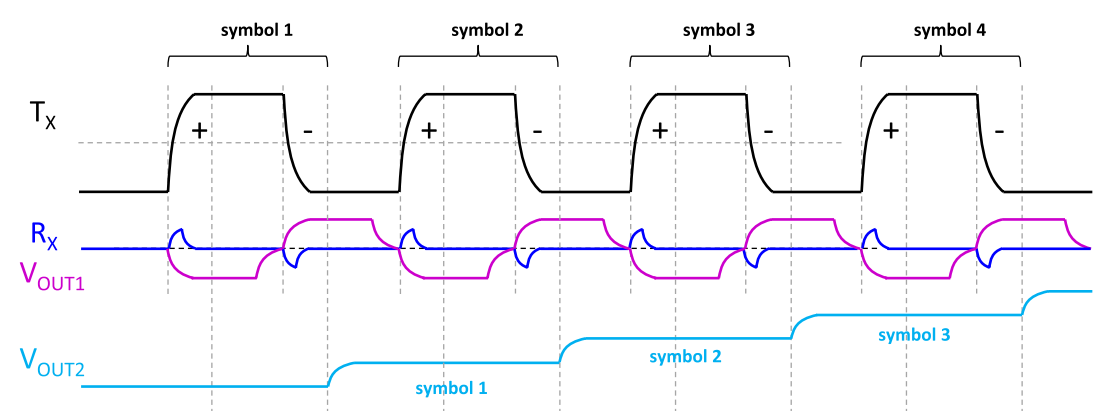

(a)

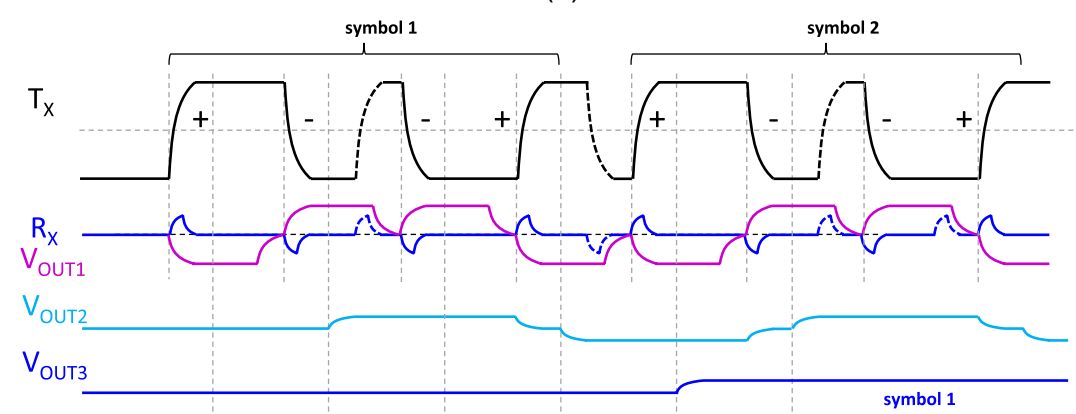

(b)

Fig. 2. (a) RZ pulse driving $+1^{\text {st }} \mathrm{CDS}$, (b) binary phase pulse driving $+1^{\text {st }} \& 2^{\text {nd }} C D S$

The normal RZ pulse driving in Tx with $1^{\text {st }} \mathrm{CDS}$ in Rx operation is as follows. First, Tx is driven by the RZ pulse signaling. CT circuit is transferring the charge in $\mathrm{C}_{\mathrm{m}}$ to $\mathrm{C}_{\mathrm{st} 1}$. And, $1^{\text {st }} \mathrm{CDS}$ circuit is sampling the output of CT circuit. The final output of $1^{\text {st }}$ CDS circuit is provided to an ADC circuit for further digital signal processing for touched data. The mode- 2 operation, the binary phase pulse driving with $1^{\text {st }} \& 2^{\text {nd }} \operatorname{CDS}$ is used, and the output of the $1^{\text {st }} \operatorname{CDS}$ is sampled by the following $2^{\text {nd }} C D S$ with $\Phi_{\mathrm{S} 3}$. The frequency of $\Phi_{\mathrm{S} 3}$ is a half of $\Phi_{\mathrm{S} 2}$.

Frequency response of two modes is summarized in as below.

Touched data and noise in mode- 1 can be expressed as

$$
S_{S}\left(@ f_{\Phi S 2}\right)+N_{S}(2 n-1)\left(@ f_{\Phi S 2}\right) \rightarrow S_{S}+N_{S}
$$

(mode-1),

where $S_{S}$ represents the touched signal, $N_{S}$ represents noise signal, and $f_{\Phi s 2}$ is the sampling frequency. The expression means touched data are obtained from sam- 
pling $\left(\Phi_{\mathrm{S} 2}\right)$ plus noise at the odd harmonics of the sampling frequency.

Touched data and noise in mode- 2 is given as

$$
S_{S}\left(f_{\Phi S 2}\right)+N_{S}\{(2 n-1) \pm 0.5\}\left(@ f_{\Phi s 2}\right) \rightarrow S_{S}+N_{S}
$$

The expression means touched data are obtained from sampling $\left(\Phi_{\mathrm{S} 2}\right)$ and noise present at $(2 n-1) \pm 0.5$ of the sampling frequency is added due to the sampling in the $2^{\text {nd }}$ CDS with $0.5 f_{\Phi s 2}$.

The proposed algorithm can reduce the noise interfered data by selecting the operating mode properly after inspecting the touched data. Fig. 3 shows a flow chart of the proposed NFIA. Tx driving frequency is set as $200 \mathrm{KHz}$ in our TSP and the sampling frequency of the CDS is set as $400 \mathrm{KHz}$. At the beginning, the system is operating in mode- 1 . If the noise frequency in the TSP is the same as the odd harmonics of the sampling frequency, the noise interferes with the normal touched data and the noise-interfered touched data is stored to a frame memory. After the system compares the noise-interfered touched data with the pre-determined valid touched data without any interference, the NFIA algorithm decides whether to stay in mode-1 or to switch into mode-2. For example, if the number of touched data point is more than the maximum valid touched data point (the maximum valid touched data point is set as 10), the system changed the operation from mode-1 to mode-2. During mode- 2 operation, the same procedure is processed for deciding whether to stay in mode- 2 or to switch back to mode-1 operation.

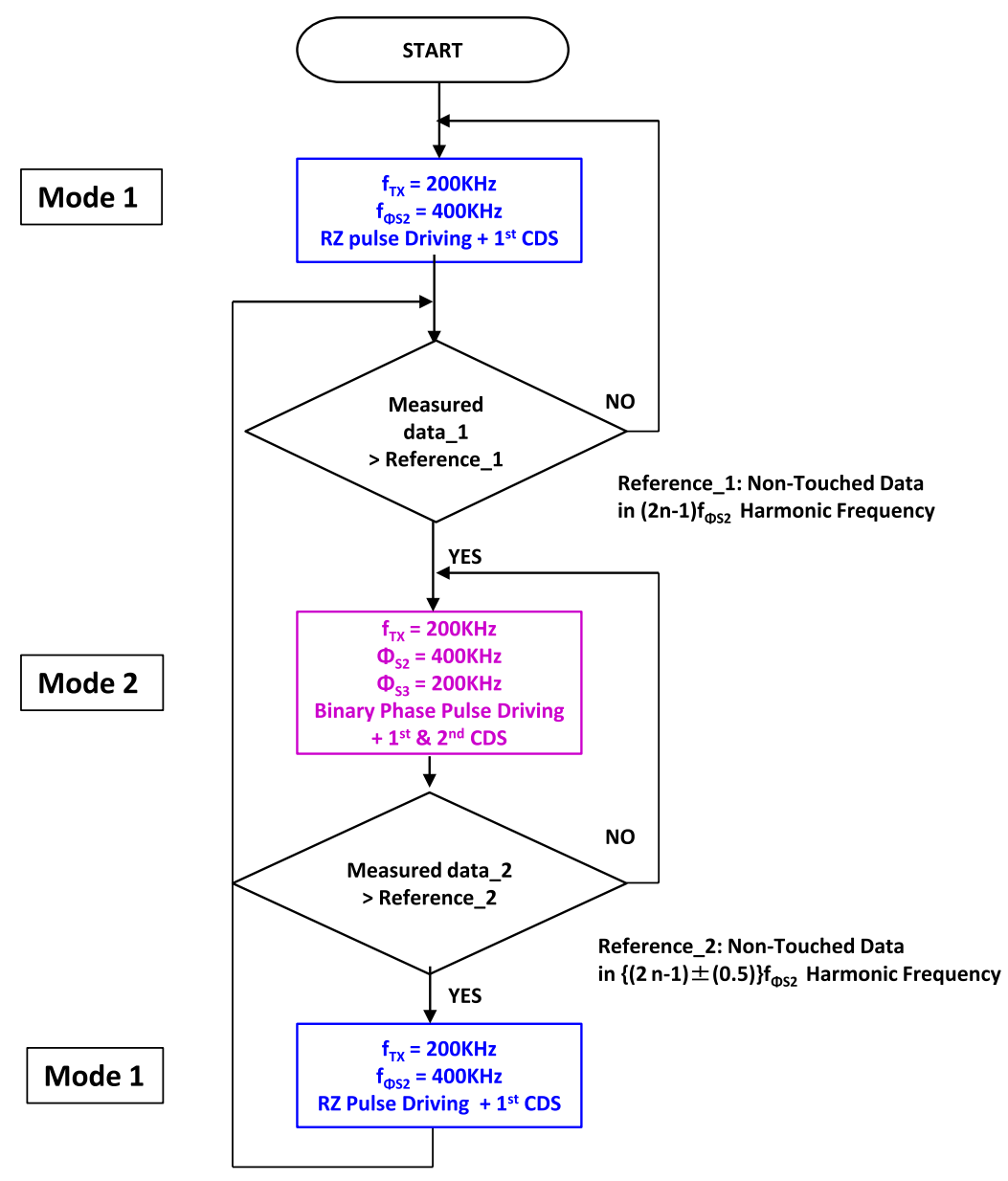

Fig. 3. Flow chart of the proposed noise frequency interference avoidance algorithm 


\section{Measurement results}

Fig. 4 shows the fabricated microphotograph. The proposed scheme has been designed and fabricated with $0.13 \mu \mathrm{m}$ CMOS technology and the total chip size of the capacitive touch controller is $4.1 \mathrm{~mm} \times 4.1 \mathrm{~mm}$. The proposed two-channel AFE occupies $370 \mu \mathrm{m} \times 160 \mu \mathrm{m}$. Thus the proposed single channel AFE occupies $29600 \mu \mathrm{m}^{2}$. The touch controller with the proposed NFIA is applied to 21.5-inch and 23-inch TSP module, respectively. The 21.5-inch TSP is composed of 40 channel transmitter (Tx) electrodes and 60 channel receiver (Rx) electrodes. Tx and Rx electrodes are connected to Tx driver and Rx sensor in the chip. In the 23-inch TSP module, $58 \mathrm{Tx}$ electrodes and $102 \mathrm{Rx}$ electrodes are implemented. Fig. 5 shows the measured results with X-axis of 52 channels in Tx driver, Y-axis of 52 points among 102 channels in Rx sensor, and the measured touch data in Z-axis. Fig. 5(a) shows the measured results of 2-point touched data in mode-1 operation with a $500 \mathrm{KHz}$ noise injection. The SNR is $43.47 \mathrm{~dB}$. At $600 \mathrm{KHz}$ noise injection. The operating mode is switched to mode- 2 by our NFIA. Fig. 5(b) shows the measured 2-point touched data in mode-2 operation with a $600 \mathrm{KHz}$ noise injection. The SNR is $43.20 \mathrm{~dB}$. Fig. 5(c) shows the measured 2-point touched data in mode-1 operation with a $700 \mathrm{KHz}$ noise injection. The SNR is $43.68 \mathrm{~dB}$. Our proposed scheme shows that noise signal is not interfered.

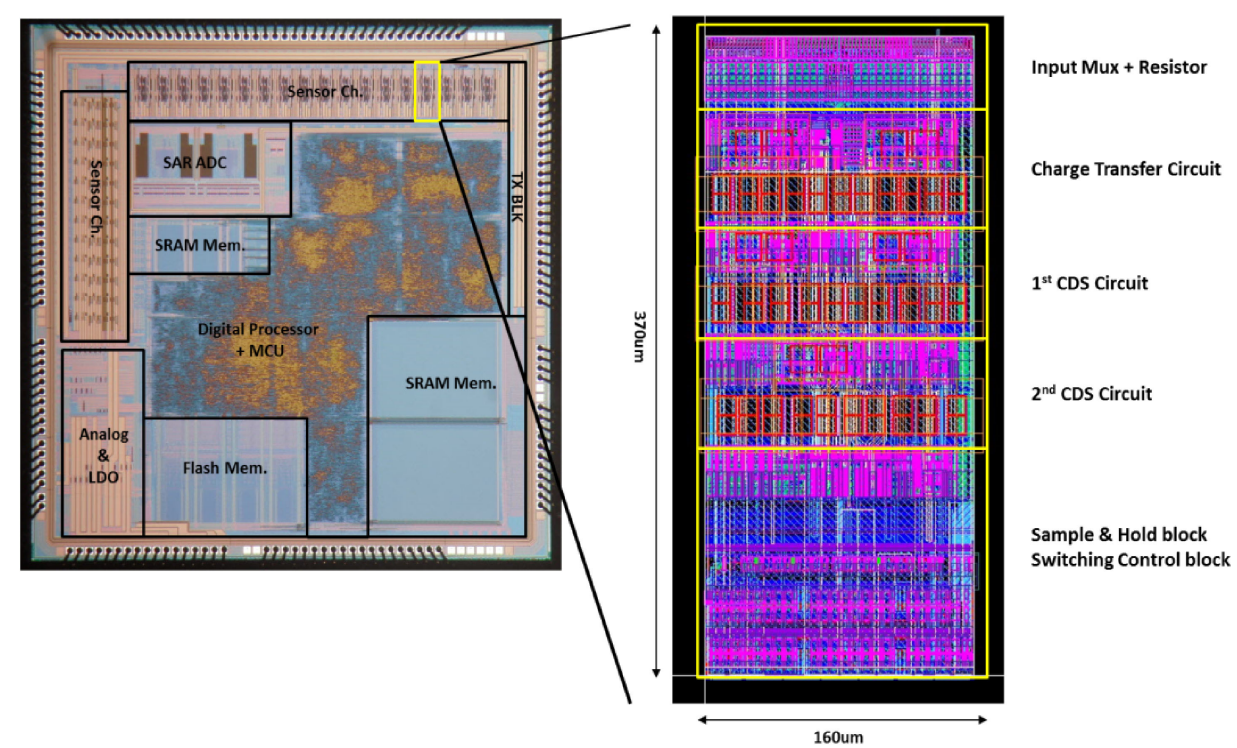

Fig. 4. Chip microphotograph \& 2-Ch. AFE layout

Measurement results and comparison with other works are summarized in Table I. The proposed NFIA technique is implemented in the touch screen controller chip, and the proposed analog front-end (AFE) block area is smaller than reference [1] and [3]. The measured results showed that the 23 inch TSP system ( Rx 2ea + Tx 1ea + MCU 1 ea) consumes $28.4 \mathrm{~mW}$ and the 21.5 inch TSP system $(\mathrm{Rx}+\mathrm{MCU}+\mathrm{Tx}$ 1ea) consumes $16.7 \mathrm{~mW}$. The measured SNR is $46 \mathrm{~dB}$ when the 


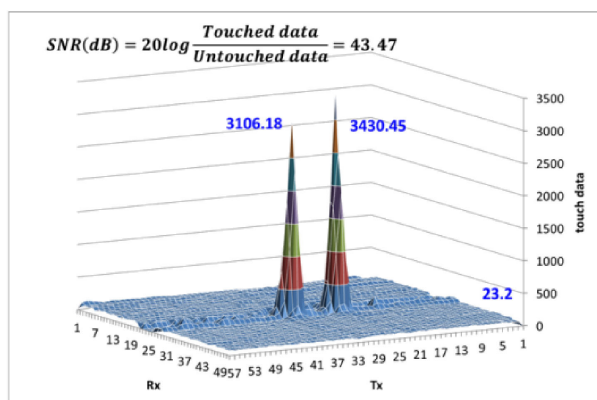

Case 1) noise $500 \mathrm{KHz}$ injection (NRZ signaling $+1^{\text {st }} \mathrm{CDS}$ )

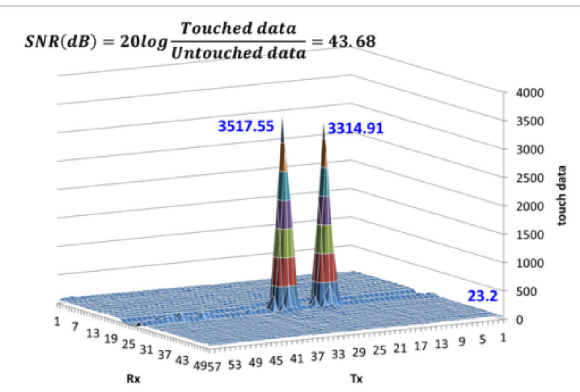

Case 3) noise $700 \mathrm{KHz}$ injection (NRZ signaling $+1^{\text {st }} \mathrm{CDS}$ )

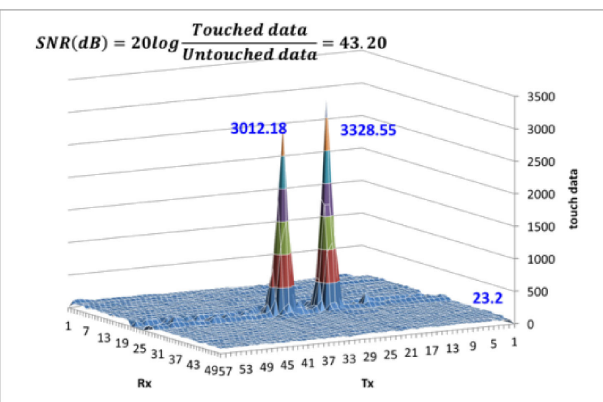

Case 2) noise $600 \mathrm{KHz}$ injection (BPSK signaling $+1^{\text {st }} \& 2^{\text {nd }} \mathrm{CDS}$ )

Fig. 5. Measurement results with different noise frequency injection case 1), 3) RZ pulse driving $+1^{\text {st }} \mathrm{CDS}$, case 2) binary phase pulse driving $+1^{\text {st }} \& 2^{\text {nd }} \mathrm{CDS}$

Table I. Performance summary and comparison with other works

\begin{tabular}{|c|c|c|c|c|}
\hline ITEM & \multicolumn{2}{|c|}{ This work } & [1] & {$[3]$} \\
\hline Size & 21.5 inch & 23 inch & $10.1 \mathrm{inch}$ & 32 inch \\
\hline TSP & $\begin{array}{l}\text { Tx: } 40 \\
\text { Rx: } 60\end{array}$ & $\begin{array}{l}\text { Tx: } 58 \\
\text { Rx: } 102\end{array}$ & $\begin{array}{l}\text { Tx: } 27 \\
\text { Rx: } 43\end{array}$ & $\begin{array}{l}\text { Tx: } 78 \\
\text { Rx: } 138\end{array}$ \\
\hline $\mathrm{Tx}$ & VCC: $3.3 \mathrm{~V}$ & VCC: $3.3 \mathrm{~V}$ & VCC: $3.3 \mathrm{~V}$ & $\begin{array}{l}\text { VCC: } 3.3 \mathrm{~V} \\
\text { (Veff.: } 52.7 \mathrm{~V})^{\dagger}\end{array}$ \\
\hline $\mathrm{Rx}$ & VCC: $3.3 \mathrm{~V}$ & VCC: $3.3 \mathrm{~V}$ & VCC: $3.3 \mathrm{~V}$ & VCC: $3.3 \mathrm{~V}$ \\
\hline SNR & $46 \mathrm{~dB} @$ finger & $42 \mathrm{~dB} @$ finger & $39 \mathrm{~dB} @$ finger & $50.8 \mathrm{~dB} @$ finger \\
\hline Power Consumption & $16.7 \mathrm{~mW}^{*}$ & $28.4 \mathrm{~mW}^{*}$ & $18.7 \mathrm{~mW}^{*}$ & $214.7 \mathrm{~mW}^{* *}$ \\
\hline Scan Rate & $120 \mathrm{~Hz}$ & $120 \mathrm{~Hz}$ & $120 \mathrm{~Hz}$ & $120 \mathrm{~Hz}$ \\
\hline Process & \multicolumn{2}{|c|}{$0.13 \mu \mathrm{m} \mathrm{CMOS}$} & $0.35 \mu \mathrm{m}$ CMOS & $0.18 \mu \mathrm{m}$ CMOS \\
\hline AFE size/ch & \multicolumn{2}{|c|}{$29,600 \mu \mathrm{m}^{2}$} & $124,000 \mu \mathrm{m}^{2}$ & $30,000 \mu \mathrm{m}^{2}$ \\
\hline
\end{tabular}

*Power consumption of Rx sensor $+\mathrm{Tx}$ driver.

${ }^{* *}$ Power consumption of $\mathrm{Rx}$ sensor $+\mathrm{Tx}$ driver + Micro-controller.

${ }^{\dagger}$ Effective Tx voltage is $52.7 \mathrm{~V}$ (255-code division driving)

\section{Conclusion}

The noise frequency interference avoidance technique for TSP controller is successfully implemented. The proposed noise frequency interference avoidance algorithm takes either RZ pulse driving with a single stage CDS or binary phase pulse driving with two cascaded CDS depending on noise frequency interference conditions. The measured results showed that the 21.5 inch TSP system consumes $16.7 \mathrm{~mW}$ and shows $46 \mathrm{~dB}$ SNR with the scan rate of $120 \mathrm{~Hz}$. 


\section{Acknowledgments}

This research was supported by Basic Science Program (NRF-2010-0020163) and by ITRC program (NIPA-2013-H0301-13-1013). The authors also thank IDEC. 